\title{
A phase-field model of solidification with convection
}

\author{
D.M. Anderson ${ }^{\text {a }}$, G.B. McFadden ${ }^{\text {b,* }}$, A.A. Wheeler ${ }^{\text {c }}$ \\ a Department of Mathematics, University of North Carolina, Chapel Hill, NC 27599, USA \\ ${ }^{\mathrm{b}}$ Mathematical and Computational Sciences Division, National Institute of Standards and Technology, Gaithersburg, MD 20899-8910, USA \\ ${ }^{\mathrm{c}}$ Faculty of Mathematical Studies, University of Southampton, Highfield, Southampton SO17 1BJ, UK \\ Received 29 October 1998; accepted 15 March 1999 \\ Communicated by H. Müller-Krumbhaar
}

\begin{abstract}
We develop a phase-field model for the solidification of a pure material that includes convection in the liquid phase. The model permits the interface to have an anisotropic surface energy, and allows a quasi-incompressible thermodynamic description in which the densities in the solid and liquid phases may each be uniform. The solid phase is modeled as an extremely viscous liquid, and the formalism of irreversible thermodynamics is employed to derive the governing equations. We investigate the behavior of our model in two important simple situations corresponding to the solidification of a planar interface at constant velocity: density change flow and a shear flow. In the former case we obtain a non-equilibrium form of the Clausius-Clapeyron equation and investigate its behavior by both a direct numerical integration of the governing equations, and an asymptotic analysis corresponding to a small density difference between the two phases. In the case of a parallel shear flow we are able to obtain an exact solution which allows us to investigate its behavior in the sharp interface limit, and for large values of the viscosity ratio. (C2000 Elsevier Science B.V. All rights reserved.
\end{abstract}

PACS: 05.70.Fh; 05.70.Ln; 81.10.Mx; 47.20.Hw

Keywords: Phase-field; Convection; Solidification; Diffuse interface

\section{Introduction}

Diffuse theories of interfaces separating two bulk phases were developed in the 19th century by Poisson [1], Gibbs [2], Maxwell [3], Rayleigh [4] and van der Waals [5]. Previously, interfaces had been modeled by Young, Laplace and Gauss as surfaces. In the latter formulation the interface is regarded as a singular surface on which associated physical mechanisms are localized and represented as boundary conditions to be applied at the surface; e.g., the notion of surface energy as an energy per unit area of the interfacial surface. This description of a phase boundary is sometimes referred to as a 'sharp interface' model and results in a so-called 'free boundary problem'. In contrast, diffuse interface theories recognize that, in reality, the interface has a finite thickness (albeit small compared with

\footnotetext{
${ }^{*}$ Corresponding author. Fax: 001-301-9904127.
} 
typical macroscopic length scales) in which physical quantities, such as density or composition, vary between their values in the adjacent bulk phases (see, e.g., [6,7]). Quantities that in the sharp interface formulation are regarded as localized to the interfacial surface are, in the diffuse interface setting, identified as being distributed within the interfacial region. For example, the surface energy of an isothermal interface is derived from the elevated Helmholtz free energy density throughout the whole interfacial region.

Diffuse interface models may be based on an extended thermodynamics involving gradients of the thermodynamic variables to account for non-local effects. Originally such theories were formulated to investigate liquids near their critical point and have subsequently been refined and developed to account for a wide range of physical situations, such as liquid crystals [8], superconductivity [9], spinodal decomposition [10,11] and ordering transitions in alloys [12-14]. Rowlinson and Widom [15] provide a thorough account of their historical development.

The phase-field model of the first-order phase transition associated with the solidification of a pure material was first proposed by Langer [16,17] and subsequently developed by a number of researchers [18-23]. Phase-field models provide an example of a diffuse interface model in which an order parameter, $\phi$, is postulated whose value indicates the phase of the system at a particular point in space and time (in this paper $\phi=1$ and $\phi=0$ denote the solid and liquid phases, respectively). Langer represented the free energy of a single-component system by a gradient energy functional of the form

$$
\mathcal{F}=\int_{V}\left\{\frac{1}{2} \epsilon^{2}|\nabla \phi|^{2}+f(\phi, T)\right\} \mathrm{d} V
$$

where $\epsilon$ is the gradient energy coefficient and $T$ is the temperature. The free energy density, $f(\phi, T)$, has a double-well structure with respect to $\phi$ in which the two local minima correspond to the solid and liquid phases. Langer proposed the following governing equations for the phase field and temperature:

$$
\begin{aligned}
& M \frac{\partial \phi}{\partial t}=-\frac{\delta \mathcal{F}}{\delta \phi}=\left\{\epsilon^{2} \nabla^{2} \phi-\frac{\partial f}{\partial \phi}\right\}, \\
& c \frac{\partial T}{\partial t}=k \nabla^{2} T+L \frac{\partial \phi}{\partial t},
\end{aligned}
$$

where $1 / M$ is a positive constant termed the mobility, $c$ is the heat capacity, $k$ the thermal conductivity and $L$ the latent heat per unit volume of the material. This phase-field formulation replaces the free-boundary problem associated with the sharp interface model of an interface by a coupled pair of non-linear reaction diffusion equations. The location of the interface is represented by the level set $\phi=1 / 2$.

The original derivation of the phase-field equations was justified by requiring the free energy of the system to decrease monotonically in time. The equation for the temperature (3) was based on a modification of the heat equation to allow a source term that accounts for latent heat production at a moving interface. Subsequently, Penrose and Fife [23] and others [24-27] applied the arguments of irreversible thermodynamics to the derivation of the phase-field equations, establishing that they are consistent with non-negative local entropy production.

The phase-field equations have been extended to allow for anisotropic surface energy by a number of authors $[20,28,29]$. In particular, Kobayashi [28] proposed that the gradient energy coefficient, $\epsilon$, may be regarded as a function of $\nabla \phi$ in order to model surface energy anisotropy, and Taylor and Cahn [30,31] suggested that the square gradient term in the free energy functional be replaced by $[\Gamma(\nabla \phi)]^{2}$, where $\Gamma(\nabla \phi)$ is a homogeneous degree one function of its argument. Wheeler and McFadden [32] showed how this formulation could be used to define a generalized form of the $\xi$-vector [33,34], which provides an elegant description of surface energy anisotropy. If we denote $\vec{p}=\nabla \phi$, then the $\xi$-vector is a homogeneous function of $\vec{p}$ of degree zero with components given by

$$
\xi_{j}(\vec{p})=\frac{\partial}{\partial p_{j}} \Gamma(\vec{p})
$$


$\vec{\xi}$ satisfies the fundamental relation [33]

$$
\mathrm{d} \Gamma(\vec{p})=\vec{\xi} \cdot \mathrm{d} \vec{p}
$$

For an isotropic surface energy, this gives $\vec{\xi}=\nabla \phi /|\nabla \phi|$.

The relation between the phase-field equations and the free-boundary formulation can be established by examining the so-called sharp interface limit of the phase-field equations. The phase-field equation has solutions in which fronts form with a width proportional to $\epsilon$ wherein $\phi$ varies between zero and one; the fronts represent interfaces. By investigating solutions of the phase-field equations in the limit $\epsilon \rightarrow 0$, Caginalp [22] showed that the governing equations of the phase-field model converge to a sharp interface model with associated boundary conditions, i.e., a free-boundary problem. By considering different distinguished limits Caginalp showed that a variety of different free-boundary problems emerge in the sharp interface limit, most of which are different forms of the classical Stefan problem, as well as the Hele-Shaw problem. Karma and Rappel [35] also examined different distinguished limits that allow more flexible interpretations of interface kinetics to be used in numerical calculations. For the anisotropic phase-field model McFadden et al. [36] obtained, in the sharp interface limit, the anisotropic version of the Gibbs-Thomson equation in two dimensions. Subsequently, Wheeler and McFadden [32] extended this analysis to three dimensions by employing the generalized $\xi$-vector.

One of the main advantages of the phase-field formulation over the free-boundary problem lies in its potential to compute realistic and complex interface shapes associated with dendritic growth. Early calculations [37] were restricted by the available computing power to simple interface geometries. Kobayashi [28] identified the importance of surface energy anisotropy for the computation of dendritic growth and exhibited computational results corresponding to both two-and three-dimensional dendrites. Subsequently, numerous workers [29,38-41] have refined the computational technique, provided numerical solutions with improved accuracy, and addressed issues related to the viability of the computational approach.

The phase-field model described above does not include coupling to the momentum equation and viscous stress tensor of classical hydrodynamics. The aim of the present paper is to formulate a phase-field model that is fully coupled to the equations of hydrodynamics in a self-consistent manner. We shall find that this requires the inclusion of an additional stress tensor associated with the diffuse interface. Wheeler and McFadden [42] showed, using Noether's theorem (see, e.g., [43]) that associated with the steady phase-field (2) there exists a second rank tensor

$$
\boldsymbol{\Xi}=\left[\frac{1}{2} \epsilon^{2}|\nabla \phi|^{2}+f\right] \boldsymbol{I}-\epsilon^{2} \nabla \phi \otimes \nabla \phi,
$$

where $\otimes$ denotes the outer product (see Appendix A) and $\boldsymbol{I}$ is the unit tensor, which satisfies the conservation law $\nabla \cdot \boldsymbol{\Xi}=0 . \boldsymbol{\Xi}$ represents the part of the stress tensor that results in capillary forces within the interfacial region. This conservation law has also been derived by Fried and Gurtin $[44,45]$ using an alternative approach employing configurational forces. The tensor $\boldsymbol{\Xi}$ is the counterpart of the capillary tensor [46] that acts as the reversible part of the stress tensor in the theory of fluids near a critical point; the irreversible part is provided by the standard viscous stress term of a Newtonian liquid.

In the original theory for a critical fluid, the density, which satisfies the continuity equation, is treated as the order parameter and appears instead of $\phi$ in the capillary tensor analogous to Eq. (6). The momentum equation, modified to include the divergence of the capillary tensor, governs the flow while an equation of state relates the pressure, temperature, and density. This original theory for a critical fluid has been extended to investigate a range of hydrodynamic phenomena including capillary waves, moving contact lines, droplets and nucleation [47]. In the context of a binary fluid, the composition may play the role of a conserved order parameter that satisfies a Cahn-Hilliard equation [10]. A variety of situations have been studied ranging from spinodal decomposition to thermocapillary flow; the review by Anderson et al. [47] and references therein provide further details. An early 
attempt to include fluid motion within a phase-field model is due to Caginalp and Jones $[48,49]$. They appended the inviscid momentum equation and the continuity equation to the phase-field model, but did not address the issues of momentum balance in the solid and capillary contributions to the stress tensor. Diepers et al. [50] have employed the methodology of two-phase fluid flow, where $\phi$ is interpreted as a solid fraction. Their model is used to study coarsening in a binary solid/liquid mixture with and without flow. Tönhardt and Amberg have also performed two-dimensional numerical studies using adaptive finite elements to study the effects of a shear flow on dendritic growth morphology [51,52].

In this paper we bring together several ideas to develop a phase-field model which allows for convection in the liquid phase. Our model has two notable aspects: first, we represent both the solid and liquid phases as Newtonian fluids in which the viscosity of the putative solid phase is specified to be much larger than that of the liquid phase. Second, the interface is ascribed an anisotropic surface energy, which is non-standard for a model which treats the two phases as Newtonian fluids. These unconventional features are in keeping with our intention to model a solid-liquid system. In order to obtain the desired viscosity variation between the phases, the viscosity is assumed to depend on the phase field, $\phi$. The anisotropic surface energy is achieved by employing the generalized $\xi$-vector formalism [42]. Unlike previous diffuse interface models, which incorporate fluid motion coupled to a conserved order parameter description [47], we adopt a non-conserved order parameter, $\phi$, in line with our aim of directly extending conventional phase field models of solidification to account for convection. This has the advantage that we may treat quasi-incompressible systems [53] in which the density of the solid and liquid bulk phases are each spatially uniform by allowing the density, $\rho$, to be a prescribed function of $\phi$.

With these assumptions we develop the irreversible thermodynamics of the model from gradient functionals for both the entropy and internal energy. We identify governing equations that are consistent with the first and second laws of thermodynamics. The quasi-incompressibility assumption, which allows the density to depend solely on $\phi$ and not the pressure, $p$, restricts the form of the thermodynamic potentials that may be employed [53]. The model comprises the compressible Navier-Stokes equations with a modified stress tensor which includes additional terms related to $\boldsymbol{E}$ as given in Eq. (6), an energy equation and a phase-field equation involving a material time derivative of $\phi$.

In order to investigate and validate this new model we examine it in some simple situations. First, we consider an isothermal planar interface at equilibrium. We show that the model recovers the classical equilibrium state in which the chemical potential and pressure are equal in each bulk phase and the changes in temperature and pressure for coexistence are related to the density and entropy mismatch of the two phases by the Clausius-Clapeyron equation. We then investigate the non-equilibrium situation in which the planar interface advances with constant velocity and an advective flow is induced in the liquid due to the density mismatch. We derive a non-equilibrium form of the Clausius-Clapeyron equation, obtain numerical solutions and conduct an asymptotic analysis for small density mismatch. Finally, we investigate the situation in which a planar interface advances with constant speed into the melt with a parallel shear flow ahead of it. We are able to obtain closed form solutions and investigate them in both the sharp-interface limit as well as the limit in which the viscosity of the solid is much greater than that in the liquid.

\section{The model}

We consider a non-isothermal system consisting of a pure material that may exist in two distinct phases. We follow the standard phase-field methodology and introduce a phase-field variable, $\phi(\vec{x}, t)$, whose value indicates the thermodynamic phase of the system as a function of time, $t$, and position, $\vec{x}$. Both phases are treated as fluids, although in the applications we will assume that one phase has a much larger viscosity and interpret it as an approximation to a solid phase. In many solidification applications, a fluid model is used for the thermodynamic 
description of the the solid phase, in that the elastic properties of the solid are ignored. We will also consider that the phase transition is first order and has an anisotropic surface energy, which is unconventional for a fluid-fluid system, but is consistent with our intention to model a solid-liquid system. We adopt the convention that $\phi=0$ denotes the liquid phase and $\phi=1$ denotes the solid phase. A solid-liquid interface is represented by a thin layer in which the phase field varies rapidly between zero and unity. The governing equations are derived by following the formalism of irreversible thermodynamics [54], as originally applied to the phase-field equations by Penrose and Fife and others [23-25]. The steady-state versions of the equations can also be obtained by appealing to variational arguments as in Ref. [42]. Derivations based on mechanical microforce balance laws, as developed by Gurtin et al. [55,56], are also possible.

\subsection{Non-equilibrium equations}

We assume that the total entropy, $\mathcal{S}$, in a material volume, $\Omega(t)$, of the system is given by

$$
\mathcal{S}=\int_{\Omega(t)}\left[\rho s-\frac{1}{2} \epsilon_{\mathrm{S}}^{2} \Gamma^{2}(\nabla \phi)\right] \mathrm{d} V
$$

where $\rho$ is the density and $s$ the entropy per unit mass. The first term in the integrand, $\rho s$, is the classical entropy density (per unit volume) and the second is a non-classical term associated with spatial gradients of the phase field. Here the gradient entropy coefficient $\epsilon_{\mathrm{S}}$ is assumed to be a constant for simplicity, and $\Gamma$ is a homogeneous function of degree unity. As we show below, the function $\Gamma$ allows for a general anisotropic surface energy of the solid-liquid interface. An isotropic surface energy results from the choice $\Gamma(\nabla \phi)=|\nabla \phi|$.

The total mass, $\mathcal{M}$, linear momentum, $\overrightarrow{\mathcal{P}}$, and internal energy, $\mathcal{E}$, associated with the material volume are assumed to have the form

$$
\begin{aligned}
& \mathcal{M}=\int_{\Omega(t)} \rho \mathrm{d} V, \\
& \overrightarrow{\mathcal{P}}=\int_{\Omega(t)} \rho \vec{u} \mathrm{~d} V, \\
& \mathcal{E}=\int_{\Omega(t)}\left[\rho e+\frac{1}{2} \rho|\vec{u}|^{2}+\frac{1}{2} \epsilon_{\mathrm{E}}^{2} \Gamma^{2}(\nabla \phi)\right] \mathrm{d} V,
\end{aligned}
$$

respectively. ${ }^{1}$ Here $\vec{u}$ is the velocity, $e$ the internal energy density (per unit mass) and $\epsilon_{\mathrm{E}}$ the gradient energy coefficient, which is assumed to be constant. The thermodynamic relations

$$
\begin{aligned}
& \mathrm{d} e=T \mathrm{~d} s+\frac{p}{\rho^{2}} \mathrm{~d} \rho+\frac{\partial e}{\partial \phi} \mathrm{d} \phi, \\
& e=T s-p / \rho+\mu,
\end{aligned}
$$

are assumed to apply locally, where $p$ is the thermodynamic pressure and $\mu$ is the chemical potential (or Gibbs free energy per unit mass).

The physical balance laws for mass, linear momentum, and internal energy are given by

$$
\frac{\mathrm{d} \mathcal{M}}{\mathrm{d} t}=0,
$$

\footnotetext{
${ }^{1}$ For simplicity we omit a possible gradient term in the density functional $\mathcal{M}$; its inclusion modifies the continuity equation and replaces the gradient Helmholtz free energy coefficient $\epsilon_{\mathrm{F}}$ in Eqs. (29) and (35) by a gradient coefficient $\epsilon_{\mathrm{K}}$ corresponding to a thermodynamic, or Kramers, potential function. This is briefly indicated in Section 2.2.
} 


$$
\begin{aligned}
& \frac{\mathrm{d} \overrightarrow{\mathcal{P}}}{\mathrm{d} t}=\int_{\delta \Omega(t)} \hat{n} \cdot \boldsymbol{m} \mathrm{d} A, \\
& \frac{\mathrm{d} \mathcal{E}}{\mathrm{d} t}+\int_{\delta \Omega(t)} \vec{q}_{\mathrm{E}} \cdot \hat{n} \mathrm{~d} A=\int_{\delta \Omega(t)} \hat{n} \cdot \boldsymbol{m} \cdot \vec{u} \mathrm{~d} A,
\end{aligned}
$$

respectively, where $\hat{n}$ is the outward unit normal to $\delta \Omega(t), \boldsymbol{m}$ the stress tensor, and $\vec{q}_{\mathrm{E}}$ the internal energy flux. The momentum balance (14) requires that the rate of change of the total momentum of the material volume results from forces acting on its boundary $\delta \Omega(t)$ (for simplicity we neglect body forces such as gravity; their inclusion is straightforward). The energy balance (15) equates the rate of change of the total internal energy of $\Omega(t)$ plus the energy flux through its boundary to the rate of work of the forces at its boundary.

In addition, the entropy balance takes the form

$$
\frac{\mathrm{d} \mathcal{S}}{\mathrm{d} t}+\int_{\delta \Omega(t)} \vec{q}_{\mathrm{S}} \cdot \hat{n} \mathrm{~d} A=\int_{\Omega(t)} \dot{s}^{\text {prod }} \mathrm{d} V,
$$

where $\vec{q}_{\mathrm{S}}$ is the entropy flux and $\dot{s}^{\text {prod }}$ is the local rate of entropy production. The second law of thermodynamics is then expressed by the requirement that $\dot{j}^{\text {prod }}$ is non-negative.

To proceed we recast the conservation laws (13)-(16) as differential equations. These are used to express the local entropy production in terms of the fluxes $\boldsymbol{m}, \vec{q}_{\mathrm{E}}$, and $\vec{q}_{\mathrm{S}}$, as well as $\mathrm{D} \phi / \mathrm{D} t$. We then identify forms for these quantities which ensure that the local entropy production is non-negative. The fluxes that result from this procedure involve both classical contributions and non-classical contributions that depend on $\nabla \phi$. In addition, we obtain an evolution equation for the phase field.

Applying the Reynolds transport theorem [57] to the mass balance law (13) gives the continuity equation in its conventional form

$$
\frac{\mathrm{D} \rho}{\mathrm{D} t}+\rho \nabla \cdot \vec{u}=0,
$$

where the material derivative of $\rho$ is denoted by $\mathrm{D} \rho / \mathrm{D} t=\partial \rho / \partial t+(\vec{u} \cdot \nabla) \rho$. Similarly, the linear momentum equation takes the form

$$
\rho \frac{\mathrm{D} \vec{u}}{\mathrm{D} t}=\nabla \cdot \boldsymbol{m},
$$

where, if $m_{j k}$ denotes the components of $m$, then $\nabla \cdot \boldsymbol{m}$ has components $\partial m_{j k} / \partial x_{j}$.

The energy equation is more complicated, and we discuss it in more detail. From Eq. (15) it follows that

$$
\int_{\Omega(t)}\left[\rho \frac{\mathrm{D} e}{\mathrm{D} t}+\rho \vec{u} \cdot \frac{\mathrm{D} \vec{u}}{\mathrm{D} t}-\nabla \cdot(\boldsymbol{m} \cdot \vec{u})+\nabla \cdot \vec{q}_{\mathrm{E}}\right] \mathrm{d} V+\frac{\mathrm{d}}{\mathrm{d} t} \int_{\Omega(t)} \frac{1}{2} \epsilon_{\mathrm{E}}^{2} \Gamma^{2}(\nabla \phi) \mathrm{d} V=0 .
$$

In Appendix A we show that

$$
\frac{\mathrm{d}}{\mathrm{d} t} \int_{\Omega(t)} \frac{1}{2} \Gamma^{2}(\nabla \phi) \mathrm{d} V=\int_{\Omega(t)} Q_{\mathrm{G}} \mathrm{d} V,
$$

where

$$
Q_{\mathrm{G}}=\nabla \cdot\left(\Gamma \vec{\xi} \frac{\mathrm{D} \phi}{\mathrm{D} t}\right)-\frac{\mathrm{D} \phi}{\mathrm{D} t} \nabla \cdot(\Gamma \vec{\xi})-\Gamma \nabla \vec{u}: \vec{\xi} \otimes \nabla \phi+\frac{1}{2} \Gamma^{2} \nabla \cdot \vec{u} .
$$

Here we have introduced the Cahn-Hoffman $\xi$-vector [33] for a diffuse interface [42] as given by Eq. (4). Hence we deduce that

$$
\rho \frac{\mathrm{D} e}{\mathrm{D} t}+\rho \vec{u} \cdot \frac{\mathrm{D} \vec{u}}{\mathrm{D} t}-\nabla \cdot(\boldsymbol{m} \cdot \vec{u})+\nabla \cdot \vec{q}_{\mathrm{E}}+\epsilon_{\mathrm{E}}^{2} Q_{\mathrm{G}}=0 .
$$


We now employ the conservation of linear momentum (18) to rewrite this equation as

$$
\rho \frac{\mathrm{D} e}{\mathrm{D} t}+\vec{u} \cdot(\nabla \cdot \boldsymbol{m})-\nabla \cdot(\boldsymbol{m} \cdot \vec{u})+\nabla \cdot \vec{q}_{\mathrm{E}}+\epsilon_{\mathrm{E}}^{2} Q_{\mathrm{G}}=0,
$$

which on using the identity

$$
\nabla \cdot(\boldsymbol{m} \cdot \vec{u})=(\nabla \cdot \boldsymbol{m}) \cdot \vec{u}+\boldsymbol{m}: \nabla \vec{u}
$$

simplifies to yield the energy equation as

$$
\rho \frac{\mathrm{D} e}{\mathrm{D} t}+\nabla \cdot \vec{q}_{\mathrm{E}}=\boldsymbol{m}: \nabla \vec{u}-\epsilon_{\mathrm{E}}^{2} Q_{\mathrm{G}} .
$$

Here $\boldsymbol{m}: \nabla \vec{u}=m_{k j} \partial u_{j} / \partial x_{k}$ (with summation over repeated indices implied). In an analogous way, the entropy balance (16) leads to the result

$$
\rho \frac{\mathrm{Ds}}{\mathrm{D} t}+\nabla \cdot \vec{q}_{\mathrm{S}}=\dot{\mathrm{s}}^{\mathrm{prod}}+\epsilon_{\mathrm{S}}^{2} Q_{\mathrm{G}} .
$$

The thermodynamic relation between $\mathrm{D} s / \mathrm{D} t$ and $\mathrm{D} e / \mathrm{D} t$ follows from Eq. (11) and is given by

$$
\frac{\mathrm{D} e}{\mathrm{D} t}=T \frac{\mathrm{D} s}{\mathrm{D} t}+\frac{p}{\rho^{2}} \frac{\mathrm{D} \rho}{\mathrm{D} t}+\frac{\partial e}{\partial \phi} \frac{\mathrm{D} \phi}{\mathrm{D} t} .
$$

The continuity equation (17) and Eqs. (21), (25) and (27) can be used to express the entropy production given by Eq. (26) as

$$
\begin{aligned}
\dot{s}^{\text {prod }}= & \frac{1}{T}\left\{\boldsymbol{m}+\epsilon_{\mathrm{F}}^{2} \Gamma \vec{\xi} \otimes \nabla \phi+\left[p-\frac{\epsilon_{\mathrm{F}}^{2}}{2} \Gamma^{2}\right] \boldsymbol{I}\right\}: \nabla \vec{u}+\frac{1}{T}\left\{\epsilon_{\mathrm{F}}^{2} \nabla \cdot(\Gamma \vec{\xi})-\rho \frac{\partial e}{\partial \phi}\right\} \frac{\mathrm{D} \phi}{\mathrm{D} t} \\
& +\nabla \cdot\left(\vec{q}_{\mathrm{S}}-\frac{\vec{q}_{\mathrm{E}}}{T}-\frac{\epsilon_{\mathrm{F}}^{2}}{T} \Gamma \vec{\xi} \frac{\mathrm{D} \phi}{\mathrm{D} t}\right)+\left(\vec{q}_{\mathrm{E}}+\epsilon_{\mathrm{E}}^{2} \Gamma \vec{\xi} \frac{\mathrm{D} \phi}{\mathrm{D} t}\right) \cdot \nabla\left(\frac{1}{T}\right),
\end{aligned}
$$

where $\epsilon_{\mathrm{F}}^{2}=\epsilon_{\mathrm{E}}^{2}+T \epsilon_{\mathrm{S}}^{2}$.

We now make the following choices for the constitutive equations for the fluxes and $\mathrm{D} \phi / \mathrm{D} t$ which ensure that $\dot{s}^{\text {prod }}$ is positive

$$
\begin{aligned}
& \boldsymbol{m}=\left[-p+\frac{\epsilon_{\mathrm{F}}^{2}}{2} \Gamma^{2}\right] \boldsymbol{I}-\epsilon_{\mathrm{F}}^{2} \Gamma \vec{\xi} \otimes \nabla \phi+\boldsymbol{\tau}, \\
& M \frac{\mathrm{D} \phi}{\mathrm{D} t}=\epsilon_{\mathrm{F}}^{2} \nabla \cdot(\Gamma \vec{\xi})-\rho \frac{\partial e}{\partial \phi} \\
& \vec{q}_{\mathrm{E}}=\tilde{k} \nabla\left(\frac{1}{T}\right)-\epsilon_{\mathrm{E}}^{2} \Gamma \vec{\xi} \frac{\mathrm{D} \phi}{\mathrm{D} t} \\
& \vec{q}_{\mathrm{S}}=\frac{\tilde{k}}{T} \nabla\left(\frac{1}{T}\right)+\epsilon_{\mathrm{S}}^{2} \Gamma \vec{\xi} \frac{\mathrm{D} \phi}{\mathrm{D} t}
\end{aligned}
$$

Here $\tau$ is the viscous stress tensor, which for a Newtonian fluid is given by $\tau=\mu\left(\nabla \vec{u}+\nabla \vec{u}^{T}\right)+\lambda(\nabla \cdot \vec{u}) I$, where $\mu$ and $\lambda$ are coefficients of viscosity, $\boldsymbol{I}$ is the unit tensor, and $M$ is a positive mobility coefficient which we take 
to be constant. A constant value for the thermal conductivity $k$ corresponds to the choice $\tilde{k}=T^{2} k$. The resulting equations of motion assume the form

$$
\begin{aligned}
& \frac{\mathrm{D} \rho}{\mathrm{D} t}=-\rho \nabla \cdot \vec{u} \\
& \rho \frac{\mathrm{D} \vec{u}}{\mathrm{D} t}=\nabla \cdot\left[\left(-p+\frac{1}{2} \epsilon_{\mathrm{F}}^{2} \Gamma^{2}\right) \boldsymbol{I}-\epsilon_{\mathrm{F}}^{2} \Gamma \vec{\xi} \otimes \nabla \phi+\boldsymbol{\tau}\right], \\
& M \frac{\mathrm{D} \phi}{\mathrm{D} t}=\epsilon_{\mathrm{F}}^{2} \nabla \cdot(\Gamma \vec{\xi})-\rho \frac{\partial e}{\partial \phi}, \\
& \rho \frac{\mathrm{D} e}{\mathrm{D} t}=\nabla \cdot[k \nabla T]+\epsilon_{\mathrm{E}}^{2} \nabla \cdot(\Gamma \vec{\xi}) \frac{\mathrm{D} \phi}{\mathrm{D} t}+\left[\left(-p+\frac{1}{2} T \epsilon_{\mathrm{S}}^{2} \Gamma^{2}\right) \boldsymbol{I}-T \epsilon_{\mathrm{S}}^{2} \Gamma \vec{\xi} \otimes \nabla \phi+\boldsymbol{\tau}\right]: \nabla \vec{u} .
\end{aligned}
$$

\subsection{Equilibrium equations}

The equilibrium form of the above governing equations with $\vec{u}=0$ admits an isothermal solution that also satisfies

$$
\begin{aligned}
& 0=\nabla \cdot\left[\left(-p+\frac{\epsilon_{\mathrm{F}}^{2}}{2} \Gamma^{2}\right) \boldsymbol{I}-\epsilon_{\mathrm{F}}^{2} \Gamma \vec{\xi} \otimes \nabla \phi\right], \\
& 0=\epsilon_{\mathrm{F}}^{2} \nabla \cdot(\Gamma \vec{\xi})-\rho \frac{\partial e}{\partial \phi} .
\end{aligned}
$$

By applying the divergence operator in (37) and using the fundamental relation (5), Eq. (37) can be reduced to the form

$$
0=\nabla p+\epsilon_{\mathrm{F}}^{2} \nabla \cdot(\Gamma \vec{\xi}) \nabla \phi .
$$

These equations also result directly from a variational formulation, which may be stated equivalently as either an entropy maximization or an energy minimization; we briefly sketch the latter argument. We temporarily include a gradient coefficient $\epsilon_{\mathrm{M}}$ in the density functional (8) to illustrate the appearance of the thermodynamic, or Kramers, potential function in the formulation; we subsequently set $\epsilon_{M}=0$ to recover the simpler model that we consider in the remainder of the paper.

We consider a convection-free equilibrium state, and set

$$
0=\delta\left(\mathcal{E}-\lambda_{\mathrm{S}} \mathcal{S}-\lambda_{\mathrm{M}} \mathcal{M}\right)=\delta \int_{V}\left[\rho e-\lambda_{\mathrm{S}} \rho s-\lambda_{\mathrm{M}} \rho+\frac{\left(\epsilon_{\mathrm{E}}^{2}+\lambda_{\mathrm{S}} \epsilon_{\mathrm{S}}^{2}-\lambda_{\mathrm{M}} \epsilon_{\mathrm{M}}^{2}\right)}{2} \Gamma^{2}(\nabla \phi)\right] \mathrm{d} V,
$$

where $\lambda_{\mathrm{S}}$ and $\lambda_{\mathrm{M}}$ are Lagrange multipliers that are introduced to account for the constraints of constant total entropy and mass. Taking variations with respect to $\delta s, \delta \rho$, and $\delta \phi$ gives

$$
\begin{aligned}
& 0=\rho e_{1}-\lambda_{\mathrm{S}} \rho, \\
& 0=e+\rho e_{\rho}-\lambda_{\mathrm{S}} 1-\lambda_{\mathrm{M}}, \\
& 0=\rho e_{\phi}-\left(\epsilon_{\mathrm{E}}^{2}+\lambda_{\mathrm{S}} \epsilon_{\mathrm{S}}^{2}-\lambda_{\mathrm{M}} \epsilon_{\mathrm{M}}^{2}\right) \nabla \cdot(\Gamma \vec{\xi}),
\end{aligned}
$$

respectively. Eq. (41) implies that the temperature $T=e_{s}$ is uniform and equal to the Lagrange multiplier $\lambda_{\mathrm{s}}$. Using the relation $e_{\rho}=p / \rho^{2}$, Eq. (42) gives that $\lambda_{\mathrm{M}}=e+p / \rho-T s=\mu$ is also constant. In Eq. (43) it 
follows that $\epsilon_{\mathrm{E}}^{2}+\lambda_{\mathrm{S}} \epsilon_{\mathrm{S}}^{2}-\lambda_{\mathrm{M}} \epsilon_{\mathrm{M}}^{2}=\epsilon_{\mathrm{E}}^{2}+T \epsilon_{\mathrm{S}}^{2}-\mu \epsilon_{\mathrm{M}}^{2}=\epsilon_{\mathrm{K}}^{2}$, where $\epsilon_{\mathrm{K}}$ is a gradient coefficient corresponding to the thermodynamic, or Kramers, potential function $\rho e-\rho T s-\rho \mu$. Setting $\epsilon_{\mathrm{M}}=0$ replaces $\epsilon_{\mathrm{K}}$ by the Helmholtz free energy coefficient $\epsilon_{\mathrm{F}}$ defined by $\epsilon_{\mathrm{F}}^{2}=\epsilon_{\mathrm{E}}^{2}+\lambda_{\mathrm{S}} \epsilon_{\mathrm{S}}^{2}$, so that Eq. (43) becomes identical to Eq. (38). We henceforth set $\epsilon_{\mathrm{M}}=0$, so that $\epsilon_{\mathrm{K}}$ reduces to $\epsilon_{\mathrm{F}}$.

The equivalence of Eqs. (43) and (39) results from the Gibbs-Duhem equation, which for constant values of $T$ and $\mu$ implies that

$$
-\nabla p=\rho e_{\phi} \nabla \phi .
$$

Alternatively, an application of Noether's theorem [43] to the translationally invariant functional $\mathcal{L}=-p+\frac{1}{2} \epsilon_{\mathrm{F}}^{2} \Gamma^{2}$ in Eq. (40), where $-p=\rho e-\rho T s-\rho \mu$, leads to a divergence-free stress tensor [42]

$$
\boldsymbol{\Xi}=\mathcal{L} \boldsymbol{I}-\frac{\partial \mathcal{L}}{\partial \nabla \phi} \otimes \nabla \phi,
$$

which is identical to the equilibrium form for $\boldsymbol{m}$ in Eq. (29) with $\boldsymbol{\tau}=0$, that also appears in Eq. (37).

Equilibrium is therefore characterized by uniform temperature and chemical potential. In contrast, there are pressure gradients in the interfacial regions where $\nabla \phi \neq 0$, although the far-field pressures in the bulk phases are equal.

\subsection{Quasi-incompressible thermodynamics}

In order to study situations in which the density in each phase is uniform, it is convenient to adopt a thermodynamic formation which does not employ the density as an independent variable, as in the model of quasi-incompressible flow considered by Lowengrub and Truskinovsky [53] and also in the work of Rooney et al. [58] on modeling thermal expansion in a Newtonian fluid. We therefore choose the pressure and temperature as independent variables, and work with a Gibbs free energy per unit mass, $g(T, p, \phi)$, which, aside from its argument, is formally identical to the chemical potential $\mu(\rho, T, \phi)$ appearing in Eq. (12). The internal energy per unit mass may then be written in the form

$$
e=g(T, p, \phi)+T s(T, p, \phi)-\frac{p}{\rho(T, p, \phi)},
$$

where we note the identities

$$
s(T, p, \phi)=-\left.\frac{\partial g}{\partial T}\right|_{p, \phi}, \quad \frac{1}{\rho(T, p, \phi)}=\left.\frac{\partial g}{\partial p}\right|_{T, \phi},\left.\quad \frac{\partial e}{\partial \phi}\right|_{s, \rho}=\left.\frac{\partial g}{\partial \phi}\right|_{p, T},
$$

where the variables which are held constant in forming the various derivatives are indicated explicitly. We prescribe the density as a function of the phase-field variable $\phi$ alone,

$$
\rho(\phi)=\rho_{\mathrm{S}} r(\phi)+\rho_{\mathrm{L}}[1-r(\phi)],
$$

where the solid and liquid densities $\rho_{\mathrm{S}}$ and $\rho_{\mathrm{L}}$ of the bulk phases are constants, and $r(\phi)$ is a smooth monotonic function that has $r(0)=0$ and $r(1)=1$; we will take $r(\phi)=\phi^{2}(3-2 \phi)$. Then, from Eq. (47) we note that since $\rho$ is independent of the pressure, the Gibbs free energy may be expressed in the form

$$
g(T, p, \phi)=g_{0}(T, \phi)+\frac{\left(p-p_{0}\right)}{\rho(\phi)},
$$

where $p_{0}$ is a reference pressure. The function $g_{0}(T, \phi)$ is assumed to have the form

$$
g_{0}(T, \phi)=\left[e_{0}-c T_{\mathrm{M}}-r(\phi) L+\frac{1}{4 a_{\mathrm{S}}} H(\phi)\right]\left(1-\frac{T}{T_{\mathrm{M}}}\right)-c T \ln \left(\frac{T}{T_{\mathrm{M}}}\right)+\frac{1}{4 a} H(\phi) .
$$


Here $e_{0}$ is a constant reference energy, the heat capacity per unit mass $c$ and latent heat per unit mass $L$ are assumed to be constant, $T_{\mathrm{M}}$ is the melting point at the reference pressure $p_{0}, a_{\mathrm{S}}$ and $a$ are constants, and $H(\phi)$ is a double-well potential, which we will assume is given by $H(\phi)=\phi^{2}(1-\phi)^{2}$. This form for $g_{0}$ is consistent with an internal energy which is a linear function of temperature, which leads to the classical heat equation in the bulk liquid [25].

The corresponding expressions for the entropy and internal energy are then

$$
\begin{aligned}
& s=\frac{1}{T_{\mathrm{M}}}\left\{e_{0}-r(\phi) L+\frac{1}{4 a_{\mathrm{S}}} H(\phi)\right\}+c \ln \left(\frac{T}{T_{\mathrm{M}}}\right), \\
& e=e_{0}+c\left(T-T_{\mathrm{M}}\right)-r(\phi) L+\left[\frac{1}{4 a_{\mathrm{S}}}+\frac{1}{4 a}\right] H(\phi)-\frac{p_{0}}{\rho(\phi)} .
\end{aligned}
$$

\section{Examples}

In this section planar solidification fronts moving with constant speed $V$ are examined. In this case, the anisotropy of the surface energy plays no role, and we assume $\Gamma(\nabla \phi)=|\nabla \phi|$. The solutions are assumed to depend only on the vertical variable $z=z^{\prime}-V t$, where $z^{\prime}$ is measured in the rest frame of the solid, and the one-dimensional solutions are time-independent functions of $z$. The velocity is measured in the rest frame, so that the velocity vanishes in the absence of driving forces. In the moving frame, the governing equations (33)-(36) can then be expressed as conservation laws for mass, horizontal and vertical components of momentum, and energy, together with the phase-field equation, as

$$
\begin{aligned}
0= & \frac{\mathrm{d}}{\mathrm{d} z}[\rho(w-V)] \\
0= & \frac{\mathrm{d}}{\mathrm{d} z}\left[\rho(w-V) u-\mu(\phi) \frac{\mathrm{d} u}{\mathrm{~d} z}\right] \\
0= & \frac{\mathrm{d}}{\mathrm{d} z}\left[\rho(w-V) w+\left\{p+\frac{1}{2} \epsilon_{\mathrm{F}}^{2}\left(\frac{\mathrm{d} \phi}{\mathrm{d} z}\right)^{2}\right\}-[2 \mu(\phi)+\lambda(\phi)] \frac{\mathrm{d} w}{\mathrm{~d} z}\right], \\
0= & \frac{\mathrm{d}}{\mathrm{d} z}\left[\rho(w-V)\left\{e+\frac{1}{2}\left(u^{2}+w^{2}\right)\right\}-\frac{\epsilon_{\mathrm{E}}^{2}}{2}(w-V)\left(\frac{\mathrm{d} \phi}{\mathrm{d} z}\right)^{2}-k(\phi) \frac{\mathrm{d} T}{\mathrm{~d} z}-\mu(\phi) u \frac{\mathrm{d} u}{\mathrm{~d} z}\right. \\
& \left.-[2 \mu(\phi)+\lambda(\phi)] w \frac{\mathrm{d} w}{\mathrm{~d} z}+w\left\{p+\frac{1}{2} \epsilon_{\mathrm{F}}^{2}\left(\frac{\mathrm{d} \phi}{\mathrm{d} z}\right)^{2}\right\}\right], \\
0= & \epsilon_{\mathrm{F}}^{2} \frac{\mathrm{d}^{2} \phi}{\mathrm{d} z^{2}}-M(w-V) \frac{\mathrm{d} \phi}{\mathrm{d} z}-\rho \frac{\partial g}{\partial \phi},
\end{aligned}
$$

respectively. Here for simplicity we consider a two-dimensional flow $\vec{u}=u \vec{i}+w \vec{k}$ with horizontal and vertical velocity components $u$ and $w$ in the $x$ and $z$ directions, respectively. The material properties $k, \mu$, and $\lambda$ are assumed to be constant in each bulk phase, depending only on $\phi$.

\subsection{Equilibrium of a planar interface}

The solution for a stationary planar interface in the absence of convection includes the conditions for thermodynamic equilibrium, and represents a special case of the discussion in Section 2.2. The sample is assumed to occupy 
the region $-\infty<z<\infty$, with $\phi(z) \rightarrow 1$ as $z \rightarrow-\infty$ and $\phi(z) \rightarrow 0$ as $z \rightarrow \infty$. The density and horizontal momentum equations are satisfied identically. The energy equation admits an isothermal solution, and the remaining equations (55) and (57) give

$$
\epsilon_{\mathrm{F}}^{2} \frac{\mathrm{d}^{2} \phi}{\mathrm{d} z^{2}}-\rho(\phi) \frac{\partial g}{\partial \phi}=0,
$$

and

$$
p=p_{\infty}-\frac{\epsilon_{\mathrm{F}}^{2}}{2}\left(\frac{\mathrm{d} \phi}{\mathrm{d} z}\right)^{2}
$$

where $p_{\infty}$ is the common value of the pressure in the bulk phases where the gradient of the phase field tends to zero. By using Eqs. (58) and (59), the derivative of $g(p, T, \phi)$ with respect to $z$ vanishes, and so represents a first integral for the system as noted in Section 2.2. Upon elimination of the pressure, Eq. (58) represents a second-order differential equation for the determination of $\phi$; some numerical examples are given below.

Equating the bulk values of the chemical potential by setting $g\left(p_{\infty}, T, 0\right)=g\left(p_{\infty}, T, 1\right)$ in Eq. (49) gives an integrated form of the Clausius-Clapeyron relation [59],

$$
\left(p_{\infty}-p_{0}\right)\left[\frac{1}{\rho_{\mathrm{S}}}-\frac{1}{\rho_{\mathrm{L}}}\right]=L\left(1-\frac{T}{T_{\mathrm{M}}}\right)
$$

which gives the dependence of the phase transition temperature on the pressure; here we recall that $T_{\mathrm{M}}$ is the melting point at the reference pressure $p_{0}$. Bulk equilibrium values for a one-dimensional planar equilibrium state are therefore completely determined if, say, the far-field value of the pressure in the solid is given, since the far-field pressure in the liquid is the same, and the Clausius-Clapeyron relation then provides the value of the temperature. Bulk values for the other thermodynamic variables follow from the known values of the temperature and pressure.

We note that LaCombe et al. [60] have proposed to take advantage of the relatively rapid response of the melting point to pressure changes in dynamic solidification studies. For example, during dendritic growth the response of the tip operating conditions to pressure-induced changes in the bulk melting point can be examined in this way. We also note that Maruyama et al. [61] have examined transitions in the kinetic growth shapes of ice $I_{h}$, from a circular disc to a hexagonal plate, in response to pressure-induced alterations of the melting point near the roughening transition.

\subsection{Density-change flow}

Here we consider the steady flow normal to a moving planar solidification front that is generated by unequal solid and liquid densities; the horizontal momentum equation is then satisfied identically. For simplicity of discussion we consider motion that is dominated by the effects of interface attachment kinetics rather than diffusion; that is, diffusion is sufficiently rapid that the system remains in thermal equilibrium, and the rate of solidification is determined by the deviation of the temperature from the equilibrium melting point. We therefore ignore the equation for energy conservation, and assume an isothermal system.

The conservation of mass equation (53) implies that

$$
\rho(w-V)=J_{\mathrm{m}},
$$

where the constant $J_{\mathrm{m}}$ represents the mass flux through the interface. For a stationary solid we have $J_{\mathrm{m}}=-\rho_{\mathrm{S}} V$, and

$$
w=-V\left(\frac{\rho_{\mathrm{S}}}{\rho(\phi)}-1\right)
$$


The flow velocity in the liquid far from the interface is then $w_{\mathrm{L}}^{\infty}=-V\left(\rho_{\mathrm{S}} / \rho_{\mathrm{L}}-1\right)$, representing a contraction flow if the solid is more dense than the liquid.

The vertical momentum equation (55) gives

$$
p-p_{\mathrm{S}}^{\infty}=-J_{\mathrm{m}} w-\frac{1}{2} \epsilon_{\mathrm{F}}^{2}\left(\frac{\mathrm{d} \phi}{\mathrm{d} z}\right)^{2}+[2 \mu(\phi)+\lambda(\phi)] \frac{\mathrm{d} w}{\mathrm{~d} z},
$$

where the constant $p_{\mathrm{S}}^{\infty}$ represents the pressure in the solid in the far field. The difference in the far-field pressures in the solid and liquid is then given by

$$
p_{\mathrm{S}}^{\infty}-p_{\mathrm{L}}^{\infty}=J_{\mathrm{m}} w_{\mathrm{L}}^{\infty}=\rho_{\mathrm{S}} V^{2}\left(\frac{\rho_{\mathrm{S}}}{\rho_{\mathrm{L}}}-1\right)
$$

which is analogous to the "vapor recoil" effect in a liquid-vapor system; i.e., a contraction flow raises the pressure in the solid relative to that in the liquid.

In addition to the jump in the bulk pressure for a moving interface, there is a jump in the chemical potential that is determined by the phase-field equation (57); this results in a generalized version of the Clausius-Clapeyron equation in which the effect of pressure on the melting point is altered by the rate of solidification. A calculation gives

$$
g_{\mathrm{L}}^{\infty}-g_{\mathrm{S}}^{\infty}=M \rho_{\mathrm{S}} V \int_{-\infty}^{\infty} \frac{1}{\rho(\phi)^{2}}\left(\frac{\mathrm{d} \phi}{\mathrm{d} z}\right)^{2} \mathrm{~d} z-\frac{V^{2}}{2}\left[\left(\frac{\rho_{\mathrm{S}}}{\rho_{\mathrm{L}}}\right)^{2}-1\right]+\frac{1}{\rho_{\mathrm{S}} V} \int_{-\infty}^{\infty}[2 \mu(\phi)+\lambda(\phi)]\left(\frac{\mathrm{d} w}{\mathrm{~d} z}\right)^{2} \mathrm{~d} z,
$$

where we recall from Eq. (62) that $w(z)$ is proportional to $V$ and vanishes if $\rho_{\mathrm{S}}=\rho_{\mathrm{L}}$. This shows that the jump in chemical potential has a term linear in the solidification rate that depends on the phase-field profile $\phi(z)$, a term proportional to $V^{2}$ involving the square of the density difference, and a viscous dissipation term proportional to $V$ (since $(\mathrm{d} w / \mathrm{d} z)^{2}$ is proportional to $V^{2}$ ). By using Eq. (64) for the pressure difference $p_{\mathrm{S}}^{\infty}-p_{\mathrm{L}}^{\infty}$, this expression can be written in the form

$$
\begin{aligned}
L\left(1-\frac{T}{T_{\mathrm{M}}}\right)-\left(p_{\mathrm{S}}^{\infty}-p_{0}\right)\left[\frac{1}{\rho_{\mathrm{S}}}-\frac{1}{\rho_{\mathrm{L}}}\right]= & M \rho_{\mathrm{S}} V \int_{-\infty}^{\infty} \frac{1}{\rho(\phi)^{2}}\left(\frac{\mathrm{d} \phi}{\mathrm{d} z}\right)^{2} \mathrm{~d} z+\frac{V^{2}}{2}\left(\frac{\rho_{\mathrm{S}}}{\rho_{\mathrm{L}}}-1\right)^{2} \\
& +\frac{1}{\rho_{\mathrm{S}} V} \int_{-\infty}^{\infty}[2 \mu(\phi)+\lambda(\phi)]\left(\frac{\mathrm{d} w}{\mathrm{~d} z}\right)^{2} \mathrm{~d} z
\end{aligned}
$$

For the special case of $\rho_{\mathrm{L}}=\rho_{\mathrm{S}}$ only the first integral on the right-hand side remains, and we recover the conventional phase-field description of kinetically controlled growth, in which $\phi(z)$ has a hyperbolic tangent profile, and the growth velocity is proportional to the product of $1 / M$ and the deviation of the temperature from its equilibrium value. The general case requires a numerical solution to determine the phase-field profile; in the following section we describe an approximate solution which is valid if the density difference between the liquid and solid phases is small.

\subsubsection{Asymptotic solution}

In this section we study the simplified case where the solid and liquid densities are nearly equal. We define the density mismatch parameter, $\delta$, by

$$
\rho_{\mathrm{S}}=\rho_{\mathrm{L}}(1+\delta)
$$


and consider the solution of Eqs. (53)-(57) in the limit $\delta \rightarrow 0$. We introduce the expansions

$$
\begin{aligned}
& \phi=\phi_{0}+\delta \phi_{1}+\cdots, \\
& V=V_{0}+\delta V_{1}+\cdots,
\end{aligned}
$$

and solve Eq. (57) for $\phi$ and $V$. The vertical component of velocity and the pressure are given by Eqs. (62) and (63), respectively. The density profile is given by Eq. (48), and the resulting vertical component of velocity is small, with $w=\mathrm{O}(\delta)$.

The $\mathrm{O}(1)$ problem for $\phi_{0}$ is given by

$$
\epsilon_{\mathrm{F}}^{2} \frac{\mathrm{d}^{2} \phi_{0}}{\mathrm{~d} z^{2}}+M V_{0} \frac{\mathrm{d} \phi_{0}}{\mathrm{~d} z}-\rho_{\mathrm{L}} \frac{\partial g_{0}}{\partial \phi}=0
$$

where $g_{0}$ is the free energy evaluated at $\phi_{0}$ (under isothermal conditions). This equation has the solution

$$
\phi_{0}(z)=\frac{1}{2}\left[1-\tanh \left(z / \ell_{\epsilon}\right)\right],
$$

where the constant $\ell_{\epsilon}$, which characterizes the thickness of the interface, is

$$
\ell_{\epsilon}=2 \epsilon_{\mathrm{F}} \sqrt{\frac{2 a_{0}(T)}{\rho_{\mathrm{L}}}} .
$$

Here

$$
\frac{1}{a_{0}(T)}=\frac{1}{a}+\frac{1}{a_{\mathrm{S}}}\left(1-\frac{T}{T_{\mathrm{M}}}\right)
$$

and the leading order interface speed is related to the temperature by

$$
M V_{0}=6 L \epsilon_{\mathrm{F}} \sqrt{2 \rho_{\mathrm{L}} a_{0}(T)}\left(1-\frac{T}{T_{\mathrm{M}}}\right)
$$

In the limit of vanishing double-well height ratio, $a / a_{\mathrm{S}} \rightarrow 0$, the velocity of the interface depends linearly on the difference in temperature from the melting temperature $T_{\mathrm{M}}$. When $T<T_{\mathrm{M}}$ the leading order interface velocity $V_{0}$ is positive (i.e. the liquid solidifies) and when $T>T_{\mathrm{M}}$ the interface velocity is negative (i.e. the solid melts).

The $\mathrm{O}(\delta)$ problem for $\phi_{1}$ is given by

$$
\mathcal{L} \phi_{1} \equiv \epsilon_{\mathrm{F}}^{2} \frac{\mathrm{d}^{2} \phi_{1}}{\mathrm{~d} z^{2}}+M V_{0} \frac{\mathrm{d} \phi_{1}}{\mathrm{~d} z}-\rho_{\mathrm{L}} \frac{\partial^{2} g_{0}}{\partial \phi^{2}} \phi_{1}=\mathcal{R},
$$

where

$$
\mathcal{R}=-M V_{1} \frac{\mathrm{d} \phi_{0}}{\mathrm{~d} z}-M V_{0}\left[1-r\left(\phi_{0}\right)\right] \frac{\mathrm{d} \phi_{0}}{\mathrm{~d} z}+\rho_{\mathrm{L}} r\left(\phi_{0}\right) \frac{\partial g_{0}}{\partial \phi}-\left[p_{\mathrm{S}}^{\infty}-p_{0}-\frac{1}{2} \epsilon_{\mathrm{F}}^{2}\left(\frac{\mathrm{d} \phi_{0}}{\mathrm{~d} z}\right)^{2}\right] r^{\prime}\left(\phi_{0}\right) .
$$

Here we have used a density profile of the form (48).

The operator $\mathcal{L}$ appearing in Eq. (75) can be written in self-adjoint form by first multiplying the equation by $\exp \left(M V_{0} z / \epsilon_{\mathrm{F}}^{2}\right)$. Upon noting that $\mathrm{d} \phi_{0} / \mathrm{d} z$ is a solution to the homogeneous problem, $\mathcal{L} \phi_{1}=0$, we deduce the solvability condition

$$
0=\int_{-\infty}^{\infty} e^{\eta z} \frac{\mathrm{d} \phi_{0}}{\mathrm{~d} z} \mathcal{R} \mathrm{d} z
$$


where $\eta=M V_{0} / \epsilon_{\mathrm{F}}^{2}$. Evaluating this integral gives

$$
M V_{1} Q_{1}\left(V_{0}\right)=-\left(p_{\mathrm{S}}^{\infty}-p_{0}\right) Q_{2}\left(V_{0}\right)+M V_{0} Q_{3}\left(V_{0}\right)+\frac{1}{2} \epsilon_{\mathrm{F}}^{2} Q_{4}\left(V_{0}\right),
$$

where $Q_{1}\left(V_{0}\right), Q_{2}\left(V_{0}\right), Q_{3}\left(V_{0}\right), Q_{4}\left(V_{0}\right)$ are the following integrals:

$$
\begin{aligned}
& Q_{1}\left(V_{0}\right)=\int_{-\infty}^{\infty} \exp (\eta \zeta)\left[\phi_{0}^{\prime}(\zeta)\right]^{2} \mathrm{~d} \zeta, \\
& Q_{2}\left(V_{0}\right)=\int_{-\infty}^{\infty} \exp (\eta \zeta) r^{\prime}\left(\phi_{0}(\zeta)\right) \phi_{0}^{\prime}(\zeta) \mathrm{d} \zeta, \\
& Q_{3}\left(V_{0}\right)=\int_{-\infty}^{\infty} \exp (\eta \zeta)\left[2 r\left(\phi_{0}(\zeta)\right)-1\right]\left[\phi^{\prime}{ }_{0}(\zeta)\right]^{2} \mathrm{~d} \zeta, \\
& Q_{4}\left(V_{0}\right)=\int_{-\infty}^{\infty} \exp (\eta \zeta) \frac{\mathrm{d}}{\mathrm{d} \zeta}\left\{\left[\phi_{0}^{\prime}(\zeta)\right]^{2} r\left(\phi_{0}(\zeta)\right)\right\} \mathrm{d} \zeta .
\end{aligned}
$$

Eq. (78) gives the the velocity correction, $V_{1}$, in terms of $V_{0}$ and $p_{S}^{\infty}-p_{0}$. Note that $V_{0}$ is related to the temperature by Eq. (74) and hence Eq. (78) can be regarded as determining the interface velocity in terms of the pressure and temperature. This relation represents the asymptotic approximation to $\mathrm{O}(\delta)$ of the non-equilibrium Clausius-Clapeyron relation given in Eq. (66). From Eq. (66) we observe that to first order in $\delta$ the only contribution from the non-equilibrium terms is due to interfacial kinetics represented by the first integral on the right-hand side.

The results of this asymptotic analysis are presented below in conjunction with the numerical results for general values of the solid and liquid densities.

\subsubsection{Numerical solution}

We next we present numerical solutions of the one-dimensional system of governing equations (53)-(57). Our aim is to solve for the phase-field profile and the solidification front velocity as the temperature and far-field pressure in the solid vary, and compare the results with our small- $\delta$ asymptotic analysis.

We solve Eq. (57) for the phase field with $w$ and $p$ given in terms of $\phi$ by Eqs. (62) and (63), respectively. The numerical procedure involves a finite difference discretization of the governing equation (57) on the domain $-L<z<L$ (where $L$ is taken to be sufficiently large so as to not influence the results of the calculations). Neumann boundary conditions $(\mathrm{d} \phi / \mathrm{d} z=0)$ are applied at $z= \pm L$ and we require that the integral of $\phi-1 / 2$ is zero, which both fixes the position of the interface near $z=0$ and provides an additional equation that allows the determination of the interface velocity for given values of the temperature and far-field pressure in the solid. The discretized system of non-linear equations is solved using Newton iteration by employing the software SNSQ [62] The program SNSQ was written by K.L. Hiebert and is based on an algorithm of Powell [63].

In Fig. 1 the solid curves display numerical results for the variation of the interface velocity with temperature for three different values of the pressure $p_{\mathrm{S}}^{\infty}$, using the material parameters for lead given in Table 1 . We put

$$
\mu(\phi)=\mu_{\mathrm{S}} r(\phi)+\mu_{\mathrm{L}}[1-r(\phi)],
$$

where $r(\phi)=\phi^{2}(3 \phi-1)$, and $\mu_{\mathrm{S}}$ and $\mu_{\mathrm{L}}$ are the dynamic viscosities of the bulk solid and liquid phases, respectively. We use $\mu_{\mathrm{S}} / \mu_{\mathrm{L}}=1$ and $\lambda(\phi)=-2 \mu(\phi) / 3$ in accordance with the Stokes hypothesis. In these calculations $\rho_{\mathrm{S}}>\rho_{\mathrm{L}}$ and so from the equilibrium form of the Clausius-Clapeyron relation (60) we expect the pressure to increase with temperature which is in agreement with the results for $V=0$ shown in this figure. In Fig. 1 we also observe that the interface velocity decreases with temperature in a roughly linear fashion at fixed pressure. The effect of changing the pressure is to shift the $V(T)$ curves while preserving their slope. This behavior is confirmed by our expression 


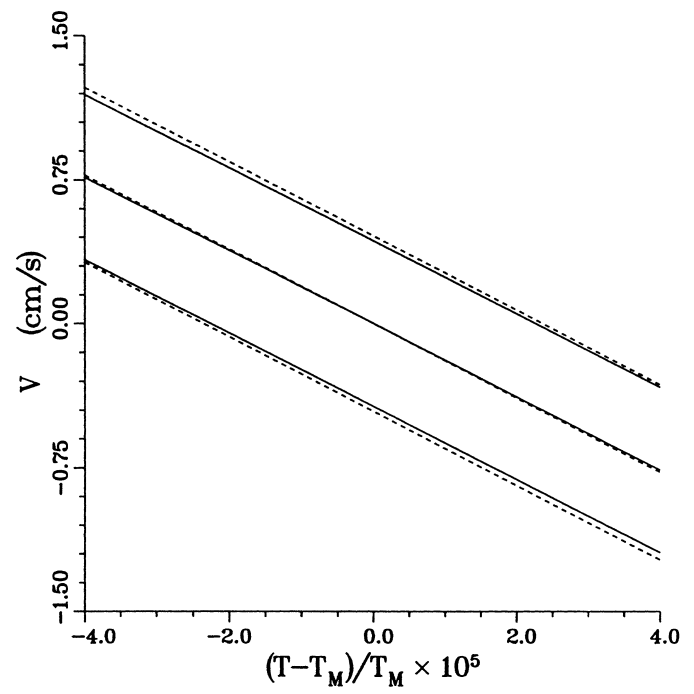

Fig. 1. A plot of the dependence of the interface velocity on temperature for three different far-field pressures in the solid. The data used is given in Table 1 and corresponds to lead. The solid curves are from the numerical solution of the governing equations and the dashed curves show the small $\delta$ asymptotic result evaluated from (78) for $\delta=0.035$. From top to bottom, the curves correspond to $p_{\mathrm{S}}^{\infty}-p_{0}=17,0$ and -17 bar, respectively.

Table 1

Properties used in calculations $[64,65]$

\begin{tabular}{llll}
\hline Property & & Value & Units \\
\hline Liquid thermal conductivity & $k_{\mathrm{L}}$ & 0.159 & $\mathrm{~J} /(\mathrm{cm} \mathrm{s} \mathrm{K})$ \\
Solid thermal conductivity & $k_{\mathrm{S}}$ & 0.297 & $\mathrm{~J} /(\mathrm{cm} \mathrm{s} \mathrm{K})$ \\
Liquid thermal diffusivity & $\kappa_{\mathrm{L}}$ & 0.108 & $\mathrm{~cm}^{2} / \mathrm{s}$ \\
Solid thermal diffusivity & $\kappa_{\mathrm{S}}$ & 0.202 & $\mathrm{~cm}^{2} / \mathrm{s}$ \\
Melting point & $T_{\mathrm{M}}$ & 600 & $\mathrm{~K}$ \\
Heat of fusion & $L_{v}$ & 256.0 & $\mathrm{~J} / \mathrm{cm}^{3}$ \\
Kinetic coefficient & $\mu$ & 33 & $\mathrm{~cm}^{3} /(\mathrm{sK})$ \\
Kinematic viscosity & $v$ & $2.43 \times 10^{-3}$ & $\mathrm{~cm}^{2} / \mathrm{s}$ \\
Liquid density & $\rho_{\mathrm{L}}$ & 10.66 & $\mathrm{~g} / \mathrm{cm}^{3}$ \\
Interface thickness & $\ell_{\epsilon}$ & $1.0 \times 10^{-8}$ & $\mathrm{~cm}$ \\
Density change & $\left(\rho_{\mathrm{S}} / \rho_{\mathrm{L}}\right)-1$ & 0.035 & - \\
\hline
\end{tabular}

for the non-equilibrium Clausius-Clapeyron equation (66), when we observe that the integrals on the right-hand side are all positive. The dashed curves in this figure represent the asymptotic approximations to these curves that are obtained in the limit of small density differences.

We also investigated the effect of increasing the viscosity ratio, $\mu_{\mathrm{S}} / \mu_{\mathrm{L}}$. We observe from the non-equilibrium Clausius-Clapeyron relation (66) that the viscous dissipation may increase without bound as the solid viscosity is increased and result in the relationship between temperature and pressure being significantly altered. However, we found from our numerical calculations that for the material parameters given in Table 1 for lead with a pressure difference $p-p_{0}=0$ this is a very weak effect; the melting temperature varied by $4 \%$ for an increase in $\mu_{\mathrm{S}} / \mu_{\mathrm{L}}$ from 1 to $10^{6}$. For a greater interface thickness of $10^{-4} \mathrm{~cm}$ the change in the melting temperature is $2 \times 10^{-3} \%$ for the same increase in $\mu_{\mathrm{S}} / \mu_{\mathrm{L}}$. 


\subsection{Shear flow}

We next consider the case of a shear flow parallel to a planar interface that propagates with a constant velocity $V$. Far from the interface, in the liquid, we assume that the component of fluid velocity parallel to the interface is $U_{\infty}$. We take a coordinate system coincident with the moving interface, which is given by the plane $z=0$. For simplicity we consider the situation where the system is isothermal, the density of the both the solid and liquid phases are equal, the surface energy is isotropic, and the dynamic viscosity has the form

$$
\mu(\phi)=\mu_{\mathrm{S}} \phi+(1-\phi) \mu_{\mathrm{L}}
$$

Under these assumptions the velocity field is given by $\vec{u}=u(z) \vec{i}$ and the governing equations (53)-(57) reduce to

$$
\begin{aligned}
& 0=\frac{\mathrm{d}}{\mathrm{d} z}\left[\rho V u+\mu(\phi) \frac{\mathrm{d} u}{\mathrm{~d} z}\right], \\
& 0=\frac{\mathrm{d}}{\mathrm{d} z}\left[p+\frac{1}{2} \epsilon_{\mathrm{F}}^{2}\left(\frac{\mathrm{d} \phi}{\mathrm{d} z}\right)^{2}\right], \\
& 0=\epsilon_{\mathrm{F}}^{2} \frac{\mathrm{d}^{2} \phi}{\mathrm{d} z^{2}}+M V \frac{\mathrm{d} \phi}{\mathrm{d} z}-\rho \frac{\partial g}{\partial \phi},
\end{aligned}
$$

where $\rho$ is the common value of the bulk densities. The phase-field equation is identical to the leading order equation for $\phi_{0}$ in the previous section. Its solution is therefore given by Eq. (71) with the requirement that the interface velocity is related to the temperature in the same manner as given in Eq. (74) (when $V$ is identified with $V_{0}$ ). The momentum equations may be integrated directly to give

$$
p(z)=p_{\infty}-\frac{\rho}{64 a_{0}(T)} \operatorname{sech}^{4}\left(\frac{z}{\ell_{\epsilon}}\right)
$$

and

$$
\frac{u(z)}{U_{\infty}}=1-A \exp \left[-\left(\frac{1+m}{2 m}\right) \frac{z}{\ell}\right]\left\{\cosh \left[\frac{z}{\ell_{\epsilon}}+\tanh ^{-1}\left(\frac{1-m}{1+m}\right)\right]\right\}^{\beta},
$$

where $\ell=\mu_{\mathrm{L}} /(\rho V)$ is the viscous boundary layer thickness in the liquid,

$$
\beta=\frac{(1-m)}{2 m} \frac{\ell_{\epsilon}}{\ell}
$$

and $m=\mu_{\mathrm{S}} / \mu_{\mathrm{L}}$. The constant of integration, $A$, is chosen to satisfy the condition that $u(-Z)=0$, where $Z>0$ :

$$
A=\exp \left[-\left(\frac{1+m}{2 m}\right) \frac{Z}{\ell}\right]\left\{\cosh \left[\frac{-Z}{\ell_{\epsilon}}+\tanh ^{-1}\left(\frac{1-m}{1+m}\right)\right]\right\}^{-\beta} .
$$

Taking the sharp interface limit, $\ell_{\epsilon} / \ell \rightarrow 0$, it is found that $u(z) \sim U_{\infty}\{1-\exp [-(z+Z / m) / \ell]\}$, for $z>0$ (in the liquid), and $u(z) \sim U_{\infty}\{1-\exp [-(z+Z) /(\ell m)]\}$, for $z<0$ (in the solid). Subsequently, taking the limit in which the viscosity of the solid is much greater than that of the liquid, $m \rightarrow \infty$, it is found to leading order in the liquid that $u(z) \sim U_{\infty}\{1-\exp (-z / \ell)\}$, and in the solid that $u(z) \sim U_{\infty}(z+Z) /(\ell m)$, i.e., a linear shear.

Thus in the sharp interface limit with the viscosity of the solid much greater than that of the liquid we recover, at leading order, the exact solution for $u(z)$ in the liquid to the underlying free boundary problem, i.e., the asymptotic boundary-layer profile $u(z)=U_{\infty}\{1-\exp (-z / \ell)\}$. In the solid phase we obtain a uniform shear flow, with a strain rate of magnitude $U_{\infty} /(2 \ell m)$. The velocity at the center of the interface $(z=0)$ is $U_{\infty} Z /(\ell m)$ and so we find that 


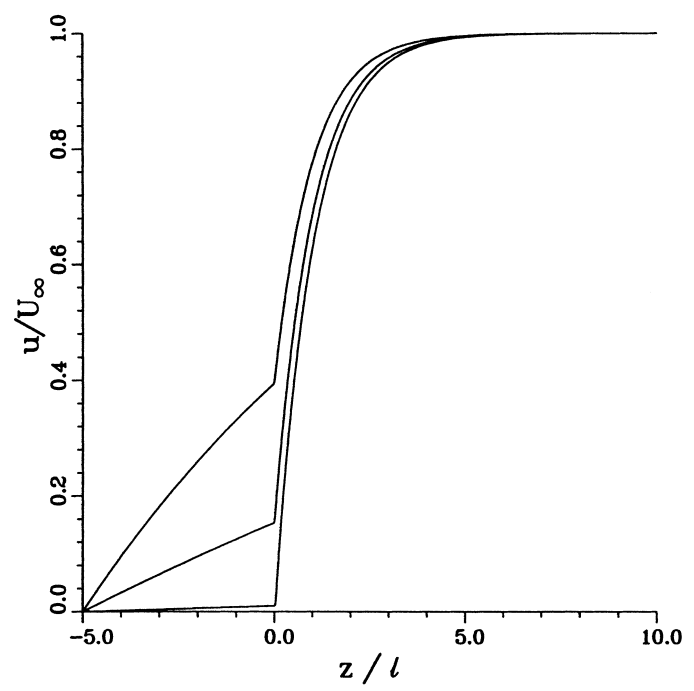

Fig. 2. A plot of $u(z)$ given by the exact solution (89) of the governing equations for $\ell_{\epsilon} / \ell=0.01$ and three different values of the viscosity ratio $\mu_{\mathrm{S}} / \mu_{\mathrm{L}}$. From top to bottom, the curves correspond to $\mu_{\mathrm{S}} / \mu_{\mathrm{L}}$ equal to 10,30 and 500.

the no-slip condition at the interface is almost satisfied, the magnitude of the error decreasing in a manner inversely proportional to $m$.

Plots of $u(z)$ for different values of $m$ with $\ell_{\epsilon} / \ell=0.01$ are given in Fig. 2. For all values of $m$ we observe a thin interfacial layer separating the linear shear in the solid from the shear flow in the liquid. We see that as $m$ increases, the flow in the solid region is suppressed and adopts a decreasingly small uniform shear while the flow in the liquid approaches the asymptotic boundary-layer profile, consonant with our asymptotic analysis. The no-slip condition at the interface, $z=0$, is satisfied with greater accuracy as $m$ increases.

The above analysis results in a closed-form solution for the specific choice (84) of the dynamic viscosity $\mu(\phi)$; other choices are, of course, possible and Eq. (84) was chosen as a compromise that maintains consistency with the form (83) used in the previous section while allowing a simple exact solution. In general, the detailed behavior of the fluid flow in the interfacial region would be expected to depend on the specific form chosen for $\mu(\phi)$. For example, interpolating by the reciprocal, as in $1 / \mu(\phi)=\phi / \mu_{\mathrm{S}}+(1-\phi) / \mu_{\mathrm{L}}$, also results in a closed-form solution. Similarly the viscous dissipation in the interface will also depend on the form of $\mu(\phi)$. This in turn will influence the degree to which the melting temperature is influenced by the ratio $\mu_{\mathrm{S}} / \mu_{\mathrm{L}}$ discussed above for a density-change flow. We note that in a related situation in which a diffuse-interface model was used to study non-equilibrium effects during directional solidification of a binary alloy [66], the details of some interfacial quantities, such as a characteristic trapping velocity, were found to be sensitive to the specific form chosen for interpolating the diffusivity through the interface; such considerations are likely to apply to the present case as well.

\section{Conclusions}

In this paper we have developed a phase-field model for the solidification of a pure material that includes convection. The model has been derived using the formalism of irreversible thermodynamics, employing gradient energy and entropy terms that incorporate the effects of capillarity within the framework of a diffuse interface model. Our solidification model has two distinctive aspects: we treat both the solid and liquid phases as Newtonian viscous fluids (with a high ratio of solid to liquid viscosity, $\mu_{\mathrm{S}} / \mu_{\mathrm{L}}$ ), and the phase boundaries are endowed with anisotropic 
surface energy by using the generalized $\xi$-vector formulation. Both features are consonant with our intention to model a solid-liquid system. Our formulation neglects elastic effects in the solid. We work with pressure and temperature as independent thermodynamic variables which permits a quasi-incompressible formulation in which the densities can be uniform in each phase. This allows us to model volume-change effects on solidification which we illustrated for the case of constant velocity isothermal unidirectional solidification. In this setting we derived a non-equilibrium form of the Clausius-Clapeyron relation which describes how the relation between pressure and temperature is modified by the motion of the interface. The ability of this two-fluid model to approximate the no-slip condition at the interface was examined by considering a model shear flow parallel to a planar interface. In this situation we found an exact solution which showed that the correction to the no-slip boundary condition is first order in the quantity $\mu_{\mathrm{L}} / \mu_{\mathrm{S}}$ for a simple model in which the viscosity depends linearly on the order parameter $\phi$.

We have focused our attention on the derivation of the model and some important test cases involving planar interfaces. In future work we plan to investigate the sharp interface limit for curved interfaces as well as use the

model as a computational vehicle for the investigation of flow effects in dendritic solidification, including the effects of buoyancy forces.

\section{Acknowledgements}

The authors are grateful for helpful discussions with W.J. Boettinger, T.J. Burns, S.R. Coriell, R.F. Sekerka, and J. Viñals. This work was conducted with the support of the Microgravity Research Division of NASA.

\section{Appendix A}

We have

$$
\begin{aligned}
\frac{\mathrm{d}}{\mathrm{d} t} \int_{\Omega(t)} \frac{1}{2} \Gamma^{2}(\nabla \phi) \mathrm{d} V & =\int_{\Omega(t)}\left[\frac{1}{2} \frac{\partial \Gamma^{2}}{\partial t}+\frac{1}{2} \nabla \cdot\left(\Gamma^{2} \vec{u}\right)\right] \mathrm{d} V \\
& =\int_{\Omega(t)}\left[\Gamma \frac{\partial \Gamma}{\partial t}+\vec{u} \cdot(\Gamma \nabla \Gamma)+\frac{1}{2} \Gamma^{2} \nabla \cdot \vec{u}\right] \mathrm{d} V \\
& =\int_{\Omega(t)}\left[\Gamma \vec{\xi} \cdot \frac{\mathrm{D}(\nabla \phi)}{\mathrm{D} t}+\frac{1}{2} \Gamma^{2} \nabla \cdot \vec{u}\right] \mathrm{d} V,
\end{aligned}
$$

where we have used the fundamental relation $\mathrm{d} \Gamma(\vec{p})=\vec{\xi} \cdot \mathrm{d} \vec{p}$, with $\vec{p}=\nabla \phi$. We note that

$$
\nabla \cdot\left(\Gamma \vec{\xi} \frac{\mathrm{D} \phi}{\mathrm{D} t}\right)=\frac{\mathrm{D} \phi}{\mathrm{D} t} \nabla \cdot(\Gamma \vec{\xi})+\Gamma \vec{\xi} \cdot \frac{\mathrm{D}(\nabla \phi)}{\mathrm{D} t}+\Gamma \nabla \vec{u}: \vec{\xi} \otimes \nabla \phi
$$

where the tensor $\Lambda=\vec{\xi} \otimes \nabla \phi$ has components $\Lambda_{j k}=\xi_{j} \partial \phi / \partial x_{k}$, and the double contraction of the tensor product is denoted by $\nabla \vec{u}: \Lambda=\partial u_{k} / \partial x_{j} \Lambda_{j k}$. This gives that

$$
\frac{\mathrm{d}}{\mathrm{d} t} \int_{\Omega(t)} \frac{1}{2} \Gamma^{2}(\nabla \phi) \mathrm{d} V=\int_{\Omega(t)}\left[\nabla \cdot\left(\Gamma \vec{\xi} \frac{\mathrm{D} \phi}{\mathrm{D} t}\right)-\frac{\mathrm{D} \phi}{\mathrm{D} t} \nabla \cdot(\Gamma \vec{\xi})-\Gamma \nabla \vec{u}: \vec{\xi} \otimes \nabla \phi+\frac{1}{2} \Gamma^{2} \nabla \cdot \vec{u}\right] \mathrm{d} V
$$




\section{References}

[1] S.D. Poisson, Bachelier, Paris, 1831.

[2] J.W. Gibbs, in: H.A. Bumstead, R.G. Van Name (Eds.), Scientific Papers of J. Willard Gibbs, Longmans, Green, and Co., London, 1928, pp. $55-371$.

[3] J.C. Maxwell, in: Scientific Papers of James Clerk Maxwell, vol. 2, Dover, New York, 1952, pp. 541-591.

[4] Lord Rayleigh, Phil. Mag. 33 (1892) 209.

[5] J.D. van der Waals, Verhandel. Konink. Akad. Weten. Amsterdam (Sect. 1), 1(8) (1893) [J.S. Rowlinson, J. Stat. Phys. 20 (1979) 197 (English trans.)].

[6] G.M. Mavrovouniotis, H. Brenner, Phil. Trans. Roy. Soc. Lond. Ser. A 345 (1993) 165.

[7] G.M. Mavrovouniotis, H. Brenner, D.A. Edwards, L. Ting, Phil. Trans. Roy. Soc. London A 345 (1993) 209.

[8] P.G. de Gennes, Mol. Cryst. Liq. Cryst. 12 (1971) 193.

[9] V.L. Ginzburg, L.D. Landau, Soviet Phys. JETP 20 (1950) 1064.

[10] J.W. Cahn, J.E. Hilliard, J. Chem. Phys. 28 (1958) 258.

[11] J.W. Cahn, Acta Metall. 9 (1961) 795.

[12] J.W. Cahn, S.M. Allen, J. Phys. (Paris) Colloque C7 (1977) C7-51.

[13] S.M. Allen, J.W. Cahn, Acta Metall. Mater. 27 (1979) 1085.

[14] R.J. Braun, J.W. Cahn, G.B. McFadden, A.A. Wheeler, Phil. Trans. Roy. Soc. Lond. Ser. A 355 (1997) 1787.

[15] J.S. Rowlinson, B. Widom, Molecular Theory of Capillarity, Clarendon Press, Oxford, 1989.

[16] J.S. Langer, Unpublished notes, 1978.

[17] J.S. Langer, in: G. Grinstein, G. Mazenko (Eds.), Directions in Condensed Matter Physics, World Scientific, Philadelphia, 1986 pp. $165-186$.

[18] G. Caginalp, in: L. Garrido (Ed.), Applications of Field Theory to Statistical Mechanics, Springer, Berlin, 1985, pp. 216-226.

[19] J.B. Collins, H. Levine, Phys. Rev. B 31 (1985) 6119.

[20] G. Caginalp, P.C. Fife, Phys. Rev. B 33 (1986) 7792.

[21] G. Caginalp, Arch. Rat. Mech. Anal. 92 (1986) 205.

[22] G. Caginalp, Phys. Rev. A 39 (1989) 5887.

[23] O. Penrose, P.C. Fife, Phys. D 43 (1990) 44.

[24] A.P. Umantsev, J. Chem. Phys. 96 (1992) 605.

[25] S.-L. Wang, R.F. Sekerka, A.A. Wheeler, B.T. Murray, S.R. Coriell, R.J. Braun, G.B. McFadden, Phys. D 69 (1993) 189.

[26] P.C. Fife, O. Penrose, Electronic J. Diff. Equations 1 (1995) 1.

[27] C. Charach, P. C. Fife, On thermodynamically consistent schemes for phase-field equations, to appear in Open Systems and Information Dynamics.

[28] R. Kobayashi, Phys. D 63 (1993) 410.

[29] A.A. Wheeler, B.T. Murray, R.J. Schaefer, Phys. D 66 (1993) 243.

[30] J.E. Taylor, Acta Metall. Mater. 40 (1992) 1475-1485.

[31] J.E. Taylor, J.W. Cahn, Physica D 112 (1998) 381.

[32] A.A. Wheeler, G.B. McFadden, Eur. J. Appl. Math. 7 (1996) 367.

[33] D.W. Hoffman, J.W. Cahn, Surf. Sci. 31 (1972) 368.

[34] J.W. Cahn, D.W. Hoffman, Acta Metall. 22 (1974) 1205.

[35] A. Karma, W.-J. Rappel, Phys. Rev. E 53 (1996) 3017.

[36] G.B. McFadden, A.A. Wheeler, R.J. Braun, S.R. Coriell, R.F. Sekerka, Phys. Rev. E. 48 (1993) 2016.

[37] G. Fix, in: A. Fasano, M. Primicerio (Eds.), Free Boundary Problems: Theory and Applications, Pitman, London, 1983, p. 580.

[38] S.-L. Wang, R.F. Sekerka, J. Comp. Phys. 127 (1996) 110.

[39] J.F. Blowey, C.M. Elliott, in: G. Buuttazzo, A. Visintin (Eds.), Motion by Mean Curvature and Related Topics, De Gruyter, New York, 1994, p. 1.

[40] A. Karma, W.-J. Rappel, Phys. Rev. E. 57 (1998) 4323.

[41] N. Provatos, N. Goldenfeld, J. Dantzig, Phys. Rev. Lett. 80 (1998) 3308.

[42] A.A. Wheeler, G.B. McFadden, Proc. Roy. Soc. Lond. Ser. A 453 (1997) 1611.

[43] H. Goldstein, Classical Mechanics, Addison-Wesley, New York, 1980.

[44] E. Fried, M.E. Gurtin, Physica D 68 (1993) 326.

[45] E. Fried, M.E. Gurtin, Physica D 72 (1994) 287.

[46] D.J. Korteweg, Arch. Néerl. Sci. Exactes Nat. Ser. II 6 (1901) 1.

[47] D.M. Anderson, G.B. McFadden, A.A. Wheeler, Ann. Rev. Fluid Mech. 30 (1998) 139.

[48] G. Caginalp, J. Jones, Appl. Math. Lett. 4 (1991) 97.

[49] G. Caginalp, J. Jones, in: M.E. Gurtin, G.B. McFadden (Eds.), On the Evolution of Phase Boundaries, IMA Series in Mathematics and Its Applications, vol. 43, Springer, New York, 1992, pp. 27-50.

[50] H.J. Diepers, C. Beckermann, I. Steinbach, in: J. Beech, H. Jones (Eds.), Solidification Processing 1997, Proc. Fourth Decennial Int. Conf. on Solid. Process, University of Sheffield, Sheffield, 1997, pp. 426-430. 
[51] R. Tönhardt, Convective effects on dendritic solidification, 1998, Ph.D. Thesis, Department of Mechanics, Royal Institute of Technology, S-100 44 Stockholm, Sweden.

[52] R. Tönhardt, G. Amberg, J. Crystal Growth 194 (1998) 406.

[53] J. Lowengrub, J. Truskinovsky, Proc. Roy. Soc. Ser. A 454 (1998) 2617.

[54] S.R. de Groot, P. Mazur, Non-Equilibrium Thermodynamics, Dover, New York, 1984.

[55] M.E. Gurtin, The nature of configurational forces, Arch. Rat. Mech. Anal. 131 (1995) 67.

[56] M.E. Gurtin, D. Polignone, J. Viñals, Math. Models Methods Appl. Sci. 6 (1996) 815.

[57] R. Aris, Vectors, Tensors, and the Basic Equations of Fluid Mechanics, Dover, New York, 1962, p. 84.

[58] F.J. Rooney, Q. Wang, S.E. Bechtel, M.G. Forest, The modeling of thermal expansion: Instability of constrained theories and constitutive limits, preprint (1998).

[59] C.H.P. Lupis, Chemical Thermodynamics of Materials, North-Holland, Amsterdam, 1983, p. 36.

[60] J.C. LaCombe, M.B. Koss, L.A. Tennenhouse, E.A. Winsa, M.E. Glicksman, J. Crystal Growth 194 (1998) 143.

[61] M. Maruyama, Y. Kishimoto, T. Sawada, J. Crystal Growth 172 (1997) 521.

[62] SLATEC Common Math Library, National Energy Software Center, Argonne National Laboratory, Argonne, IL.

[63] M.J.D. Powell, A hybrid method for nonlinear equations, in: P. Rubinowitz (Ed.), Numerical Methods for Nonlinear Algebraic Equations, Gordon and Breach, New York, 1988.

[64] S.R. Coriell, M.R. Cordes, W.J. Boettinger, R.F. Sekerka, J. Crystal Growth 49 (1980) 13.

[65] G.H. Rodway, J.D. Hunt, J. Crystal Growth 112 (1991) 554.

[66] N.A. Ahmad, A.A. Wheeler, W.J. Boettinger, G.B. McFadden, Phys. Rev. E 58 (1998) 3436. 